\title{
Sodium binding sites and permeation mechanism in the NaChBac channel: a Molecular Dynamics study
}

\author{
Carlo Guardiani, ${ }^{\dagger}$ Philip M. Rodger, ${ }^{\ddagger}$ Olena A. Fedorenko, ${ }^{\S}$ Stephen K. \\ Roberts, $^{\S}$ and Igor A. Khovanov*,, $\uparrow$ \\ School of Engineering, University of Warwick, Coventry CV4 7AL United Kingdom, \\ Department of Chemistry, University of Warwick, Coventry CV4 7AL, Centre for \\ Scientific Computing, University of Warwick, Coventry CV4 7AL United Kingdom, and \\ Division of Biomedical and Life Sciences, Lancaster University, Lancaster, UK \\ E-mail: i.khovanov@warwick.ac.uk \\ Phone: +44 (0) 24765 22600. Fax: +44 (0) 2476418922
}

\begin{abstract}
$\mathrm{NaChBac}$ was the first discovered bacterial sodium voltage dependent channel, yet computational studies are still limited due to the lack of a crystal structure. In this work a poreonly construct built using the NavMs template was investigated using unbiased Molecular Dynamics and Metadynamics. The Potential of Mean Force (PMF) from the unbiased run features four minima, three of which correspond to sites IN, CEN and HFS discovered in NavAb. During the run the Selectivity Filter (SF) is spontaneously occupied by two ions and frequent access of a third one is often observed. In the innermost sites IN and CEN, $\mathrm{Na}^{+}$is fully hydrated by six water molecules and occupies an on-axis position. In site HFS sodium interacts with a glutamate and a serine from the same subunit and is forced to adopt an off-axis placement. Metadynamics simulations biasing one and two ions, show an energy barrier in the SF that prevents single-ion permeation. An

\footnotetext{
*To whom correspondence should be addressed

${ }^{\dagger}$ School of Engineering

${ }^{\ddagger}$ Department of Chemistry

ICentre for Scientific Computing

$\S$ Division of Biomedical and Life Sciences, Lancaster
} University, Lancaster, UK
\end{abstract}

analysis of the permeation mechanism was performed both computing minimum energy paths in the axial-axial PMF and through a combination of Markov State modeling and Transition Path Theory. Both approaches reveal a knockon mechanism involving at least two, but possibly three ions. The currents predicted from the unbiased simulation using Linear Response Theory are in excellent agreement with singlechannel patch-clamp recordings.

\section{Introduction}

Multicellular organisms rely on voltagedependent cation channels for the onset and rapid propagation of electrical signals triggering key cellular events such as muscle contraction or neurosecretion. Voltage dependent sodium channels (Navs) for example, are responsible for the rising branch of the action potential while the repolarizing phase results from the opening of potassium channels. Eukaryotic voltagegated sodium and calcium channels are large monomeric proteins consisting of four homologous domains each containing a voltage-sensor subdomain encompassing helices S1-S4 and a pore subdomain comprising helices S5-S6 and the intervening P-loop. ${ }^{1,2}$ 
The importance of these channels is testified by the wide range of channelopathies arising from function impairing mutations or autoimmune reactions. Consistent with the distribution of ion channels throughout the human body, ion channel defects have been implicated in a wide variety of diseases, including epilepsy, migraine, hypertension, cardiac arrhythmia, asthma, and cancer. ${ }^{3}$

The large size and complexity of these proteins has so far prevented a full structural characterization. As an example, the most detailed 3D-structure of a Nav is a $19 \AA$ resolution cryo-electron microscopy image where not even secondary structural elements are discernible. ${ }^{4}$ The smaller and simpler prokaryotic sodium channels are thus proving to be valuable model compounds for understanding their eukaryotic counterparts. The first sodium channel to be identified, NaChBac (Fig 1) from Bacillus halodurans, ${ }^{5}$ is a homotetrameric protein whereby each subunit shows the same structural organization of the domains of eukaryotic Navs. Since its discovery, NaChBac architecture revealed puzzling similarities and differences with other ion channels. Even though NaChBac is homotetrameric like potassium channels, its selectivity filter (SF) is not delimited by the backbone carbonyl groups of the TVGYG sequence but by the side-chains of the conserved sequence TLESWAS. Thus, despite being $\mathrm{Na}^{+}$-selective, $\mathrm{NaChBac}$ shows the signature FxxxTxExW sequence of eukaryotic Calcium channels. Moreover, site-directed mutation experiments ${ }^{6}$ showed that serine to aspartate mutations in the TLESWAS sequence make the channel Calcium selective.

Currently, the molecular basis of selectivity and the permeation mechanism of potassium channels is very well characterized. ${ }^{7}$ In potassium channels the carbonyl groups of the TVGYG sequence define four binding sites S1-S4 that are occupied by either a $\mathrm{K}^{+}$ion or a water molecule. The channel is so narrow that completely dehydrated potassium ions and water molecules must adopt a single-file arrangement and are obliged to move in a concerted way. The ion movement causes a shift from the simultaneous occupation of sites S1, S3 to the occupation of sites $\mathrm{S} 2$, S4. The permeation mechanism in $\mathrm{NaChBac}$ can be expected to be completely different since the channel is sufficiently wide to accommodate a fully hydrated $\mathrm{Na}^{+}$ion so that the movement of ions and water may be uncorrelated and not occur in single-file. Some clue can be gained from the structure of a NaChBac homologue, NavAb from Arcobacter butzleri. The analysis of the crystal structure led Payandeh et $a l^{8}$ to postulate the existence of three sodium binding sites, IN, CEN and HFS at the level of the Threonines, Leucines and Glutamates of the selectivity filter respectively. This prediction was then confirmed by a number of computational studies on the NavAb system. ${ }^{9,11-14}$ However, there is no reason why this finding should be considered as a general rule and the number and position of the binding sites might be somewhat different in other sodium channels.

Another intriguing problem concerns the number of ions required in the SF for permeation. Whereas molecular dynamics studies ${ }^{11-14}$ have considered mainly a two-ion mechanism of conduction, a recent high resolution structure of the sodium NavMs channel ${ }^{10}$ reports 3 ions inside the selectivity filter. Simulation studies on NavAb ${ }^{11-14}$ showed the existence of a $3-5 \mathrm{kcal} / \mathrm{mol}$ energy barrier in the $\mathrm{SF}$ that might prevent the passage of an ion unless it is electrostatically kicked by at least one other ion so that a knock-on mechanism would be operating. Based on sequence similarity, it could be speculated that this kind of scenario also applies to the $\mathrm{NaChBac}$ system, but so far no direct experimental or computational testing has been performed. In particular, not only the existence but also the height of the barrier is a critical parameter. While a modest barrier could delay permeation of a single ion without preventing it, a very high barrier would make the single-ion mechanism impossible. A final issue deserving investigation is the hydration and coordination pattern of $\mathrm{Na}^{+}$ions in the SF. This is an extremely critical point since it may affect the selectivity of the channel. For instance, Corry and Thomas ${ }^{11}$ showed that, in $\mathrm{NavAb}, \mathrm{Na}^{+}$selectivity arises from the inability of $K^{+}$to fit between a plane of glutamate 
residues with the preferred solvation geometry, which involves water molecules bridging between the ion and carboxylate groups.

In this paper we present a combined molecular dynamics/metadynamics study of the $\mathrm{NaChBac}$ channel that addresses these issues. Since a crystal structure of $\mathrm{NaChBac}$ channel is not available, an homology model was developed and used in the simulations. The template we used for model building was the recently crystallized structure of NavMs channel. ${ }^{21}$ This protein shows much higher sequence identity (46\%) with $\mathrm{NaChBac}$ with respect to previously used ${ }^{20}$ templates like the Kv1.2/2.1 chimera (PDB ID: 2R9R; ) and the Kv1.2 channel (PDB ID: 3LUT;) both featuring about $23 \%$ sequence identity. Using this approach we were able to generate a structural model of the Pore Domain with wide open selectivity filter and activation gate which lends itself well to permeation studies.

This homology model was then embedded in a solvated bilayer and submitted to a long unbiased simulation. The resulting PMF showed four main minima, three of which correspond to sites IN, CEN and HFS of NavAb. When the PMF was computed from a metadynamics ${ }^{16}$ simulation with axial bias on a single ion in the SF, however, only a very deep minimum corresponding to site CEN could be found. This clearly shows the inability of a single ion to overcome the energy barrier inside the SF. In fact, when the metadynamics was repeated biasing two ions in the SF, we attained a PMF in good agreement with the one yielded from the unbiased simulation. The permeation mechanism was analyzed with two different methodologies. In the first approach we used the unbiased trajectory to build a Markov model and then we applied Transition Path Theory to identify the dominant permeation paths. ${ }^{17}$ The second strategy relied on tracing a Minimum Energy Path on the axial-axial PMF from 2D-metadynamics using a variant of the Nudged Elastic Band algorithm. ${ }^{14}$ The permeation paths predicted by the two methods are consistent with each other and they reveal a knock-on mechanism. Our results are validated through comparison with patch-clamp record- ings showing excellent agreement between simulated and experimental currents.

\section{Materials and Methods}

\section{$2.1 \quad$ Homology Modelling}

A structural model of the pore-only construct of $\mathrm{NaChBac}$ was built using as a template the NavMs channel from the marine bacterium Magnetococcus sp (strain MC-1) that was recently crystallized ${ }^{15}$ in the fully open conformation at $2.9 \AA$ resolution (PDB ID: 4F4L). The alignment with $\mathrm{NaChBac}$ reveals a sequence identity of $46 \%$ which makes NavMs an excellent template. The modeling was performed using the MODELLERv9.15 package. ${ }^{23}$ The $\mathrm{NaChBac}$ sequence was first aligned with the NavMs template using a variable gap penalty function that places gaps in solvent exposed regions rather than in secondary structure elements, thus significantly reducing alignment errors. Figure SF3 of the Supporting Information shows the alignment of $\mathrm{NaChBac}$ with NavMs and the NavAb homologous channel. Based on the NaChBac/NavMs alignment, a structural model was then built from each of the four subunits of the NavMs crystal. Figure SF4 of the Supporting Information shows the overlay of $\mathrm{NaChBac}$ model with the crystal structures of the NavMs template and the homologous NavAb channel. The structures show an excellent degree of superposition except for the C-terminal end of helix S6 which is related to the level of opening of the activation gate. This feature is better appreciated in Figure SF5 of the Supporting Information offering views from the intracellular and extracellular side of spacefilling representations of $\mathrm{NaChBac}$, NavMs and NavAb. It can be noted that while the Selectivity Filter on the extracellular side is wide open in all the three channels, the activation gate on the cytoplasmic side is open only in NavMs and in the derived model of $\mathrm{NaChBac}$, while it is closed in the NavAb channel. 

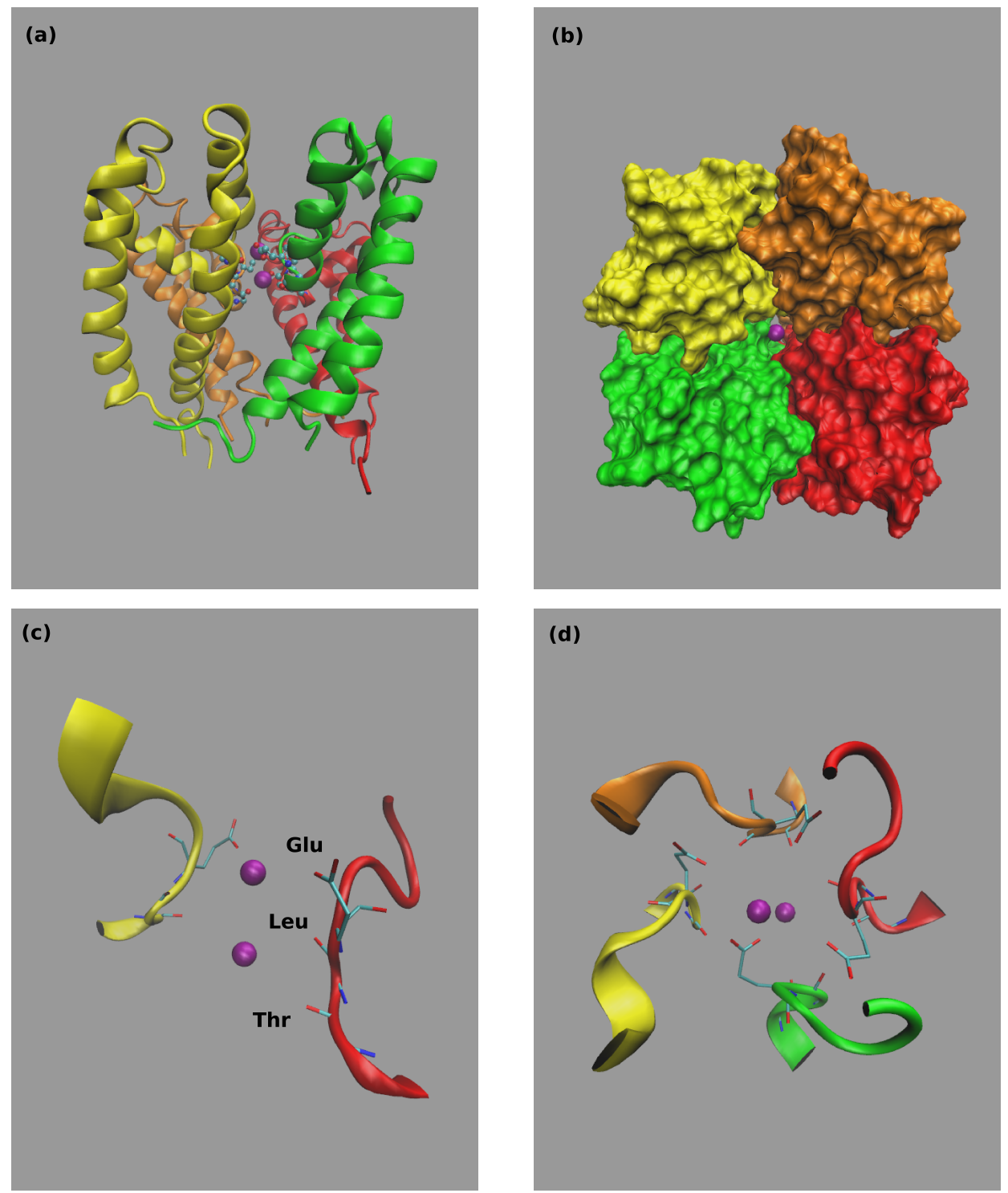

Figure 1: Structure of NaChBac channel. (a) Side view of the pore-only construct of NaChBac. Each of the four identical subunits comprizes transmembrane helices S5 and S6 as well as a linking region including the Turret Loop, P1-helix, the Selectivity Filter and P2-helix. The Glutamates and the backbone of Threonines and Leucines of the TLESWAS sequence of SF are shown in a ball-and-stick representation. The SF is occupied by two $\mathrm{Na}^{+}$ions in sites CEN and HFS, the most stable conformation emerging from our simulations. (b) Top view of a space filling representation of NaChBac. In our homology model the SF is wide open. (c) Side view of SF. For the sake of clarity only the first and third chain are portrayed. The Thr, Leu and Glu residues respectively involved in sites IN, CEN and HFS are shown in ball-and-stick representation. (d) Top view of the SF. Note the arrangement of the four glutamates to form the EEEE-ring of site HFS. 


\subsection{Unbiased simulations}

The NaChBac homology model was embedded in a bilayer comprizing 248 molecules of 1palmitoyl-2-oleoyl-sn-glycero-3-phosphocholine (POPC) and solvated by 15730 water molecules. We added 164 sodium ions and 156 chloride ions so as to neutralize the charge of the channel and reach a final concentration $[\mathrm{NaCl}]=0.5 \mathrm{M}$. Overall the system comprized 87122 atoms. All simulations were performed with the NAMD $2.11 \mathrm{~b} 2^{24}$ suit of programs (which allowed to run about $2.5 \mathrm{~ns} /$ day on 32 cores) using the ff $12 \mathrm{SB}^{26}$ force field for the protein and the Lipid14 force-field ${ }^{25}$ for the phospholipids. The system first underwent 10000 steps of conjugate gradient minimization. During equilibration harmonic restraints were applied to non-hydrogen atoms of the protein backbone and side-chains as well as to the phospholipid heads. An harmonic restraint was also applied to the didedral angle formed by Carbons 8, 9, 10, 11 of oleoyl acid and to the improper dihedral $C_{1}-C_{3}-C_{2}-O_{2}$ involving the three carbons of the glycerol unit and the hydroxyl oxygen linked to its central carbon. The equilibration was organized in six stages whereby the costraints were gradually released. The values of the force constants used in the six stages can be found in Table ST1 of the Supporting Information. The production run was carried out in the isothermal isobaric ensemble for 100 ns. Pressure was kept at 1 atm by the Nose-Hoover Langevin piston method while temperature was kept at $300 \mathrm{~K}$ by coupling to a Langevin thermostat with damping coefficient of $1 \mathrm{ps}^{-1}$. Long-range electrostatic interactions were evaluated with the smooth particle mesh Ewald algorithm. For the short-range non-bonded interactions, we used a cutoff of $12 \AA$ with a switching function at $10.0 \AA$. The integration time step was $2 \mathrm{fs}$, and the bonds between hydrogen and heavy atoms were fixed to eliminate the most rapid oscillatory motions. The procedure for the calculation of the PMF from the unbiased simulation is detailed in the Supporting Information.

\subsubsection{Metadynamics simulations}

The 1D-metadynamics simulation was started from a conformation pre-equilibrated for 1 ns in the NPT ensemble with one ion in the SF at the level of the EEEE-ring. The biased collective variable was defined as the z-component of the distance vector between the ion in the SF and the center of mass $(\mathrm{COM})$ of the $\mathrm{C}_{\alpha} \mathrm{s}$ of the four glutamates of the EEEE-ring. The space explored by the $z$ reaction coordinate was restricted to lie within the range $[-10: 10] \AA$ by $100 \mathrm{kcal} / \mathrm{mol} / \AA^{2}$ walls. The transversal mobility of the ion was also restricted by the same means within a circle of radius $4.0 \AA$ centered on an axis parallel to the $z$-axis passing through the center of mass of the $\mathrm{C}_{\alpha} \mathrm{s}$ of the glutamates of the EEEE-ring. Energy Gaussians with height of $0.005 \mathrm{kcal} / \mathrm{mol}$ and width of $0.25 \AA$ were deposited every 0.4 ps. In order to avoid over-filling artifacts, we used welltempered metadynamics ${ }^{27}$ with a virtual temperature parameter $\Delta T=1697 \mathrm{~K}$. The choice of the $\Delta T$ parameter was based on a preliminary standard metadynamics run (data not shown) which allowed to estimate the height of the energy barriers at about $E^{\dagger}=4.0 \mathrm{kcal} / \mathrm{mol}$. The virtual temperature parameter was then chosen to satisfy the relation $k_{B}(T+\Delta T)=E^{\dagger}$. The sampling was improved by applying a spherical harmonic restraint from the COM of the EEEEring to prevent the remaining $\mathrm{Na}^{+}$ions from the outer bulk from entering the SF during the long sampling. The restraint was implemented through the tclforces module of NAMD (including periodic boundary conditions) according to

$$
V\left(r_{i}\right)= \begin{cases}0 & \text { if } r_{i}>r_{c u t} \\ \frac{1}{2} K\left(r_{i}-r_{c u t}\right)^{2} & \text { if } r_{i} \leq r_{c u t}\end{cases}
$$

with $r_{c u t}=10 \AA$ and $K=20 \mathrm{kcal} / \mathrm{mol} / \AA^{2}$. The total length of the sampling slightly exceeded $100 \mathrm{~ns}$ (102 ns). The 2D-metadynamics simulation was started from a typical configuration from the NPT trajectory with two ions in the SF at the level of the LLLL-ring and EEEE-ring. The simulation protocol was the same as in the 1D-metadynamics except that the virtual temperature parameter $\Delta T$ was set 
to $2697 \mathrm{~K}$ to assure the overcoming of all energy barriers while the height of the energy gaussians was increased to $0.3 \mathrm{kcal} / \mathrm{mol}$ to speed up convergence. The simulation was run for 260 ns. The calculation of the projected axial PMF from the axial-axial 2D-PMF is illustrated in the Supporting Information where we also give an account of the modified Nudged Elastic Band algorithm and of the use of Markov State Model in conjunction of Transition Path Theory to identify the permeation mechanism.

\subsubsection{Markov State Modelling and Transition Path Theory}

Markov State Modelling is based on a large number of short simulations started from randomly chosen intermediate states. The idea is that the assembly of these small trajectories should yield a high number of instances of the long pathway from the initial to the final state. A Markov State Model is then built using the states yielded by a clustering of the concatenation of the short trajectories. The transition probabilities between couples of states are then used to compute the bottleneck flux of each of the possible pathways connecting the initial and final states. The dominant pathways are then identified as those with the maximal bottleneck flux. This approach was successfully employed by Noè et $a l^{17}$ to determine the folding mechanism of the PinWW domain and by Choudhary et $a l^{22}$ to determine the permeation pathway of ATP across the VDAC1 channel. Further details can be found in Supporting Information.

\subsection{Current-Voltage curves}

Current-Voltage curve in $\mathrm{NaChBac}$ were attained using the collective diffusion model introduced in $\operatorname{Ref}^{35}$ where the time-course $Q(t)$ of the net charge transported across the channel at equilibrium is considered as an unbiased random walk. The channel region is defined as the space between the two planes of equation $z=z_{1}$ and $z=z_{2}$ such that its length is $L_{z}=z_{2}-z_{1}$. Since in the stationary state the current across each $z$-plane must be identical, there is considerable freedom in the choice of the channel region. In our case we considered the motion of ions in the Selectivity Filter region setting $z_{1}=-4.5 \AA$ and $z_{2}=16.5 \AA$. The net charge transported in the time interval $\Delta t$ between two consecutive frames of the trajectory is

$$
\Delta Q=\sum_{z_{1} \leq z_{i} \leq z_{2}} \frac{e_{i} \Delta z_{i}}{L_{z}}
$$

where the sum runs over all ions $i$ such that $z_{1} \leq z_{i} \leq z_{2}$, and $\Delta z_{i}$ is the axial displacement of the ion in the time interval $\Delta t$. The trajectory $Q(t)$ can then be attained by cumulating all the contributions $\Delta Q: Q(t)=\sum_{t_{i} \leq t} \Delta Q\left(t_{i}\right)$. Based on diffusion theory, for sufficiently long times, the mean square displacement of the charge $<Q^{2}(t)>$ is expected to grow linearly with a slope proportional to the diffusion coefficient $D_{Q}:<Q^{2}(t)>=2 D_{Q} t+$ const (Fig SF6 of Supporting Information). Exploiting Linear Response Theory it can be shown that the steady current induced by a small constant voltage $V$ can be computed as $I_{\text {steady }}=D_{Q} V / k_{B} T$. Using such an approach the linear region of an I- $\mathrm{V}$ curve can be computed based on the spontaneous ion fluctuations at equilibrium, in the absence of any applied electric field. More details on the collective diffusion model are provided in the Supporting Information.

\subsection{Electrophysiology}

$\mathrm{NaChBac}$ channel activity was recorded using the patch-clamp technique applied to Chinese Hamster Ovary (CHO) cells transiently transfected with pTracer-CMV2 expressing NaChBac (for further details see Supporting Information). Pipette solution contained (in $\mathrm{mM}$ ) 492 Na-gluconate, $5 \mathrm{NaCl}, 10$ EGTA, and 10 HEPES, pH 7.4 (adjusted by $3 \mathrm{mM} \mathrm{NaOH}$; $[\mathrm{Na}]$ total $=500 \mathrm{mM}$ ) and standard bath solution contained (in $\mathrm{mM}$ ) $497 \mathrm{NaCl}$, and 10 HEPES, pH 7.4 (adjusted with $3 \mathrm{mM} \mathrm{NaOH}$; $[\mathrm{Na}]$ total $=500 \mathrm{mM})$. Results were analysed using Clampfit 10.1 software (Molecular Devices, Inc.) and figures were constructed using OriginPro 8 (OriginLab Corporation). Data are presented as means SEM (n), where $\mathrm{n}$ is the num- 
ber of independent experiments

\section{$3 \quad$ Results}

\subsection{Unbiased simulation}

The homology model of the pore-only construct of $\mathrm{NaChBac}$, embedded in a solvated bilayer was simulated for $100 \mathrm{~ns}$ in the NPT ensemble. After $1 \mathrm{~ns}$ a single sodium ion already occupies the selectivity filter at the level of the four glutamates of the SF. As shown in Fig 2, after 5 ns a second ion enters the SF just above the EEEE-ring where it interacts with the glutamates and serines of the TLESWAS sequence. The entrance of the second ion is made possible when the first sodium moves to a second binding site formed by the carbonyls of the four leucines of the TLESWAS sequence. The trajectory then shows several transient entering events of a third ion. Three longer-lived threeions arrangements can be observed after 35, 65 and 90 ns respectively, when the first sodium ion moves moves from the LLLL-carbonyl ring to the TTTT-ring, while the second ion still remains in the EEEE-ring but it moves down along the channel axis. Fig 2 shows that water provides most of the oxygen atoms coordinating the $\mathrm{Na}^{+}$ions. At variance with potassium channels where the $\mathrm{K}^{+}$ion can flow through the channel only once it is stripped of its hydration shell, in the $\mathrm{NaChBac}$ channel, sodium ions can cross the SF retaining a significant portion of their hydration shell. Fig 2 also shows that the water content of the SF is correlated to the number of sodium ions in the filter region. In fact, the peaks in the number of water molecules correspond to the maxima of the plot of sodium counts in the SF.

In order to determine the distribution of the sodium ions inside the channel and to estimate the height of the energy barriers encountered during the diffusion process, we computed a Potential of Mean Force (PMF) as a function of the position of the ions along the channel axis. The PMF profile, shown in Fig 3, shows the
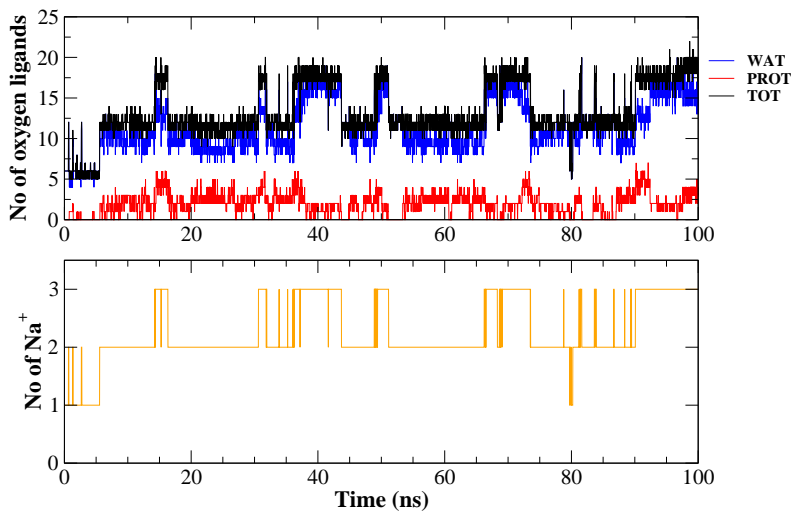

Figure 2: Occupancy of Selectivity Filter. Bottom Panel: time course of the number of $\mathrm{Na}^{+}$ ions in the SF. Top Panel: number of oxygen atoms interacting with the sodium ions in the SF. Blue line: water oxygens; red line: protein oxigens; black line: total oxygens. The selectivity filter was identified as the region along the channel axis in the range $-10 \leq z \leq 14 \AA$. The coordination analysis was performed using a cutoff distance of $3.2 \AA$.

existence of four minima. The deepest minimum in $7<z<10 \AA$ corresponds to the side chains of the glutamates of the EEEE-ring. The second main minimum in $3<z<7 \AA$ identifies a binding site at the level of the backbone carbonyl groups of the four Leucines of the TLESWAS sequence of the selectivity filter. The third minimum $(0<z<3 \AA)$ is higher in energy with respect to the first two and is thus scarcely populated. It is located at the level of the backbone carbonyls of the Threonines of the SF. The first three minima thus correspond to binding sites HFS, CEN and IN predicted by Catterall and coworkers ${ }^{8}$ in NavAb. Our PMF shows the existence of a fourth highenergy minimum, $\mathrm{S} 4$, in the $10<z<12 \AA$ region that corresponds to the side-chain of the Serine adjacent to glutamate in the TLESWAS sequence of the SF.

The error on the PMF was derived by dividing the trajectory in 4 25-ns blocks and computing the standard deviation of the free energy in each block. The errors normalized to average values in sites IN, CEN, HFS and S4 are 26\%, 13\%, $11 \%$ and $12 \%$ respectively. The most notable difference is that the relative error in the high- 
energy site IN is about twice as large as the errors in the other binding sites. This pattern suggests that site IN binds the $\mathrm{Na}^{+}$ions more weakly than the other sites thus giving rise to wider fluctuations in the occupancy.

Considering the pattern of occupancy during the trajectory, the PMF calculation was repeated using only the configurations with 2 or 3 ions in the SF. Since the SF is occupied by two ions in $70 \%$ of the frames, it is not surprising that the 2-ion PMF is almost identical to the all-ion PMF. The inset of Fig 3 shows a comparison of the PMFs computed using all ions, 2 ions and 3 ions. This plot gives some insight of the physical role of the low-energy sites IN and S4. In the case of site IN for instance, in the 2-ion PMF the IN minimum becomes shallower and almost disappears with respect to the allion PMF. On the other hand, in the 3-ion PMF the IN minimum becomes extremely deep. A similar pattern also occurs in the case of minimum S4, even if the differences between the all-ion and 2-ion PMFs in this region are really tiny. This pattern shows that sites IN and S4 are mainly populated in 3-ion configurations whereas in the 2-ion arrangements sodium ions mostly occupy sites CEN and HFS. This is confirmed by direct analysis of the trajectory. Sites IN and S4 are populated in $21 \%$ and $29 \%$ of the 2 -ion configurations respectively, while they account for $74 \%$ and $88 \%$ of the 3-ion configurations. The most populated 2-ion configurations are CEN-HFS (50\%) and CEN-S4 (27\%). Fig 3 also shows that in the 3 -ion PMF the CEN minimum becomes deeper than it is in the 2-ion and all-ion PMF. This pattern can be explained considering that the most frequent 3ion arrangement is IN-CEN-S4 (50\%). The second most frequent arrangement is CEN-HFS-S4 (26\%) explaining why also the HFS minimum in the 3-ion PMF is deeper than its counterparts in the 2-ion and all-ion PMFs.

In order to characterize the state of hydration and coordination of sodium ions in the different binding sites, in Fig 4 we plot the average number of oxygen atoms interacting with sodium ions in transversal bins with height of

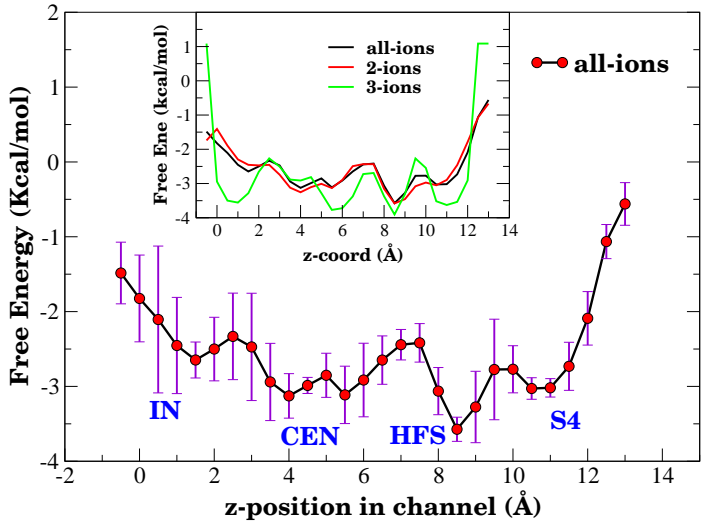

Figure 3: Potential of Mean Force of the $\mathrm{Na}^{+}$ ion in the Selectivity Filter of NaChBac channel. In the inset the PMF computed using all frames of the trajectory (black curve) is compared with the PMFs computed using only the configurations with 2 ions (red curve) or 3 ions (green curve) in the Selectivity Filter.

$2.0 \AA$ along the axis of the channel. Outside the $0-18 \AA$ range sodium is fully solvated by six water molecules. Inside this range, the channel narrows and the number of coordinating waters decreases while more coordinating oxygens are provided by the protein. Sites IN and CEN correspond to the 0-6 $\AA$ interval. In this region sodium does not directly interact with protein oxygens. In site HFS, corresponding to the 8$10 \AA$ range, sodium interacts with four waters, and two residues, a glutamate and a serine, often provided by the same subunit of the protein. Finally, in site S4, corresponding to the 10$12 \AA$ region, sodium has a $40 \%$ probability of interacting with one or two serines, and a $60 \%$ probability of being fully hydrated by water. The fluctuations in the number of coordinating oxygens is quantified by the error computed as the standard deviation of the coordination numbers of trajectory frames sampled every 20 ps to avoid correlations. A detailed analysis of these fluctuations can be found in the Supporting Information. The coordination pattern has an influence on the on- or off-axis placement of the ion. This issue can be investigated using a two-dimensional PMF as a function of the axial position $z$ and the distance $r$ from the channel axis. This PMF, illustrated in Fig 5 shows two 
main minima. The first minimum, corresponding to the axial range $0<z<6 \AA$ and the radial interval $0<r<1 \AA$, can be mapped to sites IN and CEN. Since in these regions, $\mathrm{Na}^{+}$ does not directly interact with the protein, it will adopt a central, on-axis placement. The second minimum of the 2D-PMF occupies the 7.0-10.0 $\AA$ interval along the axis at a radial distance of 2.0-4.5 $\AA$ from the channel axis so that it corresponds to the HFS minimum. Since in this region sodium directly coordinates two residues of the same chain, it is forced to occupy an off-axis position.

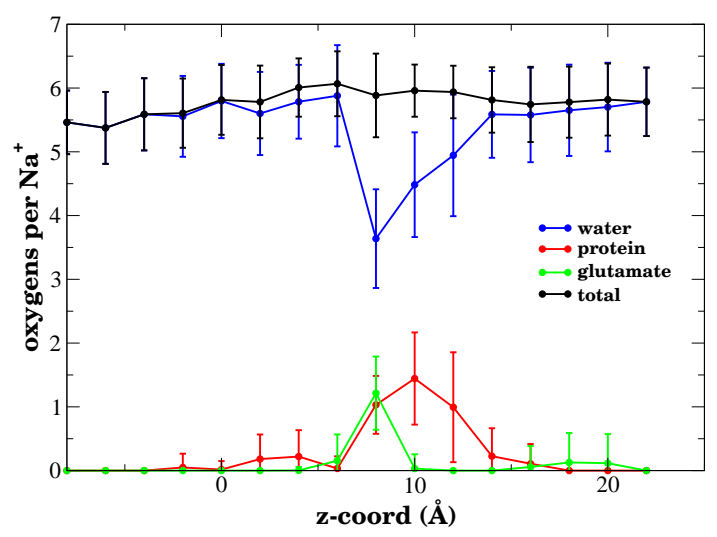

Figure 4: Coordination number for $\mathrm{Na}^{+}$in the $\mathrm{NaChBac}$ channel. Blue line: number of coordinating oxygen ions provided by water; magenta line: coordinating oxygens contributed by the glutamates of the EEEE-ring; red line: oxygens contributed by other protein residues; black line: total number of coordinating oxygens.

\subsection{Metadynamics simulations}

In order to yield a more accurate estimate of the free energy landscape of sodium ions inside the NaChBac channel, we performed metadynamics simulations. As in the case of the unbiased NPT run, also the metadynamics simulation was started from a pre-equilibrated conformation with a single ion in the SF at the level of

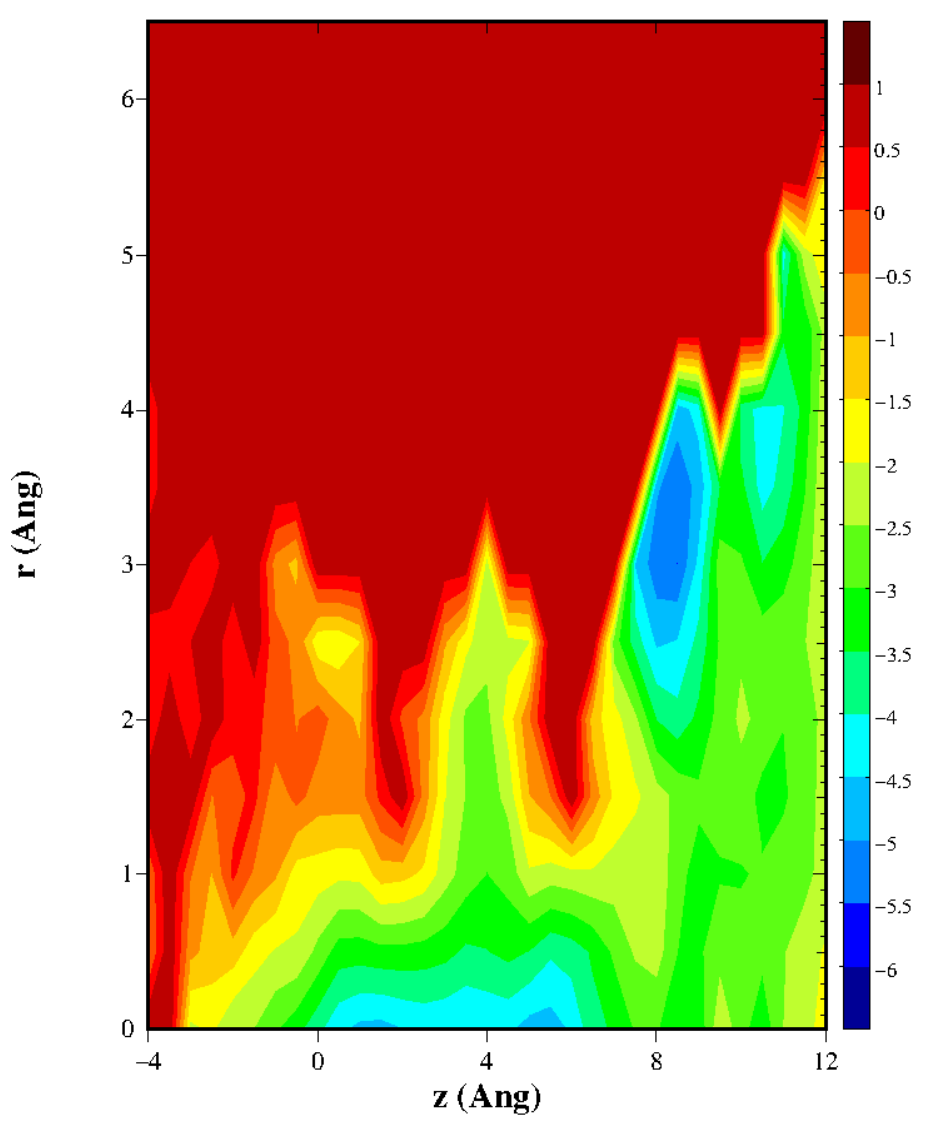

Figure 5: Radial-axial free energy plots of sodium in the $\mathrm{NaChBac}$ channel. Contour levels are at intervals of $0.5 \mathrm{kcal} / \mathrm{mol}$. 
the EEEE-ring. The biased collective variable was defined as the z-component of the distance vector between the ion in the $\mathrm{SF}$ and the center of mass $(\mathrm{COM})$ of the $C_{\alpha} \mathrm{s}$ of the four glutamates of the EEEE-ring. The total length of the sampling slightly exceeded $100 \mathrm{~ns}$ (102 ns). The Potential of Mean Force generated by the metadynamics run is shown in Fig 6 . In the inset of the Figure we show the PMF profile computed at intervals of $10 \mathrm{~ns}$. It can be noted that, as the simulation progresses, the curves become closer and closer to one another which is the signature of the approach to convergence. It can also be noted that, at variance with the PMF profile computed in the unbiased simulation (Fig 3), the metadynamics PMF features a single very deep minimum instead of four. Since the axial position of the COM of the EEEE-ring is at about $5.5 \AA$, the $[-0.5: 1.5] \AA$ range of the metadynamics PMF minimum can be mapped to the $[5.0: 7.0] \AA$ range of the plot in Fig 3 , which correspond to the upper part of the CEN binding site. The mismatch with the predictions of the unbiased simulation is presumably due to the fact that while in metadynamics we constrained the system to simulate a single ion in the SF, in the unbiased simulation the SF is spontaneously occupied by at least two ions during most of the trajectory.

If this working hypothesis is correct, then the PMF generated by a metadynamics simulation with axial biasing of two ions in the SF should produce a PMF profile more in agreement with the one yielded by the unbiased simulation. The simulation was started from a frame of the unbiased run representing a typical arrangement of the ions in the SF with a $\mathrm{Na}^{+}$in site CEN and another sodium in site HFS. The bidimensional PMF reported in Fig 7 shows two main energy basins in a symmetrical position with respect to the diagonal. In the top-left basin the first sodium ion, whose axial position defines the $z_{1}$ collective variable, occupies the lower part of the SF, while the second ion, related to the $z_{2}$ variable, is located in the upper part of the SF. In the bottom-right basin, on the other hand, the position of the two ion

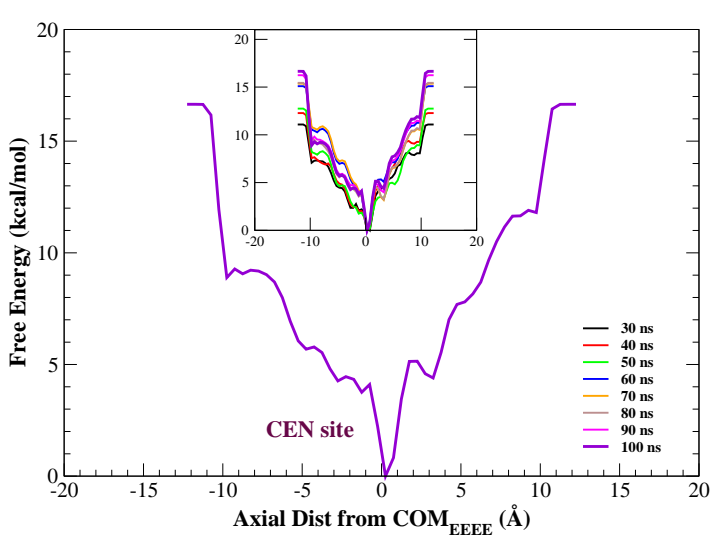

Figure 6: Potential of Mean Force of the $\mathrm{Na}^{+}$ ion in the NaChBac channel yielded by $100 \mathrm{~ns}$ of well-tempered metadynamics with axial bias of a single sodium ion in the selectivity filter. Inset: time evolution of the PMF at 10 ns intervals. The closer and closer similarity oif the curves reveals the approach to convergence.

is reversed. Since the two ions have identical physical properties, the exchange of their position does not affect the free energy thus justifying the symmetry of the contour plot.

The axial-axial PMF can be projected so as to yield a PMF as a function of the axial distance $z$ of a biased ion (irrespective of its identity) from the center of mass of the EEEEring. The projected axial PMF reported in Fig 8 is characterized by two deep minima separated by a barrier of about $3 \mathrm{kcal} / \mathrm{mol}$ in agreement with the simulations of Stock et $a l^{14}$ on the NavAb channel. Also in this case, in order to compare the position of these minima with the ones appearing in the unbiased PMF (Fig 3) it is necessary to perform a translation of $5.5 \AA$ along the $z$-axis. After this operation the first minimum, corresponding to the [4.0:6.5] A range, overlaps extensively with the CEN minimum ([3.0:7.0] $\AA$ range) of the unbiased PMF. The second minimum, extending over the $[7.5: 10.0] \AA$ range, identifies almost perfectly with the HFS minimum ([7.0:10.0] $\AA$ range) found in the unbiased simulation. The high-energy minima IN and S4 of the unbiased PMF have disappeared. 


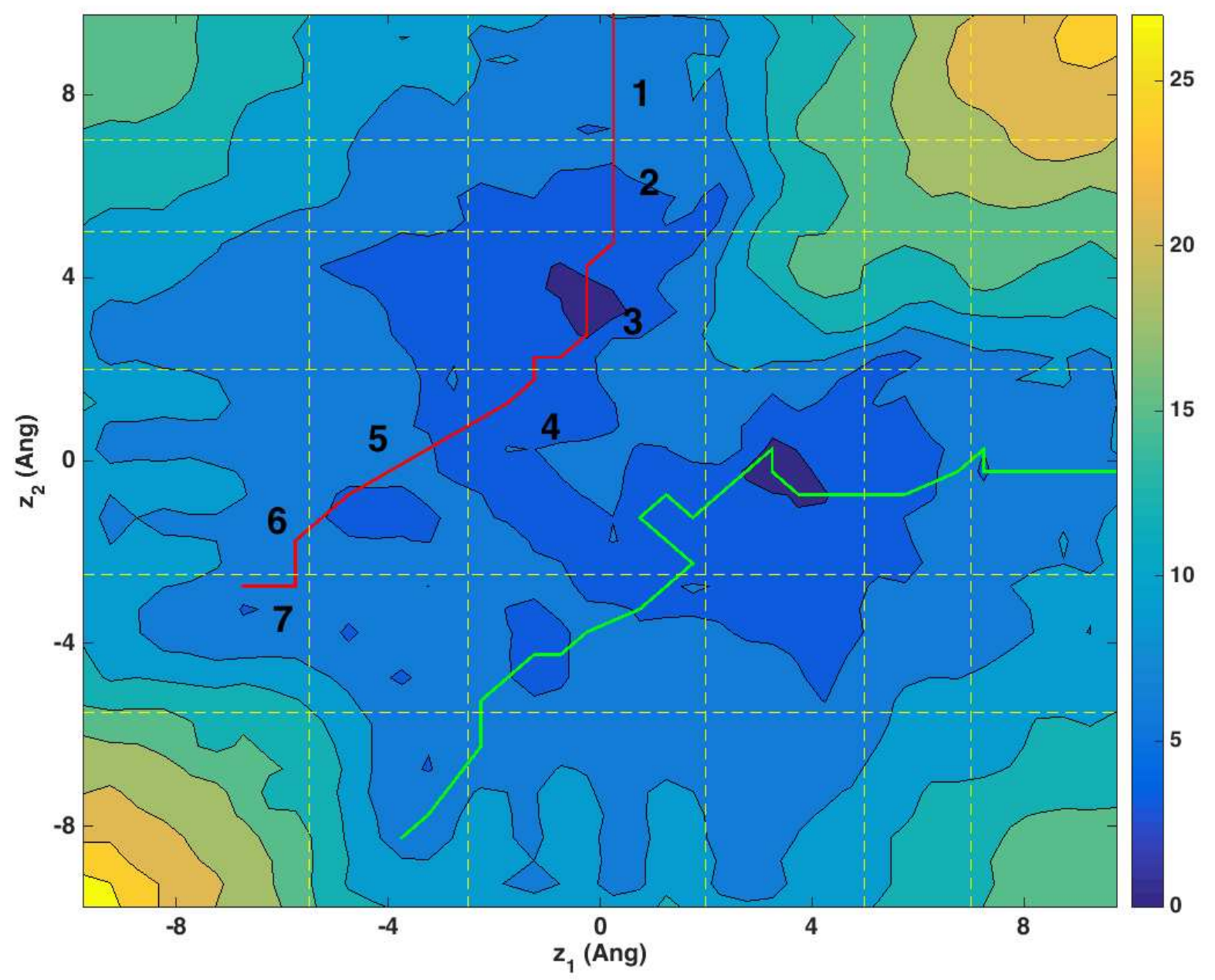

Figure 7: Axial-axial PMF of $\mathrm{Na}^{+}$computed through a 260 ns metadynamics simulation biasing two ions in the SF. The $z_{1}$ and $z_{2}$ coordinates represent the axial distance between the biased ions and the center of mass of the Glutamates of the SF. The free energy is expressed in $\mathrm{kcal} / \mathrm{mol}$ and contour lines correspond to $3 \mathrm{kcal} / \mathrm{mol}$ intervals. The red and green lines correspond to Minimum Energy Paths of permeations computed through a variant of the Nudged Elastic Band method. The yellow dashed lines represent the boundaries of the binding sites. Central Cavity: $[-10:-5.5] \AA$; site IN: $[-5.5:-2.5] \AA$; site CEN: $[-2.5: 2.0] \AA$; site HFS: $[2.0: 5.0] \AA$; site S4: $[5.0: 7.0] \AA$; External region: $[7.0: 11.0] \AA$. The numbers refer to the states visited by the two ions during permeation. 1: (CEN,Ext); 2: (CEN, S4); 3: (CEN, HFS); 4: (CEN, CEN); 5: (IN, CEN); 6: (CC,CEN); 7: (CC,IN). 


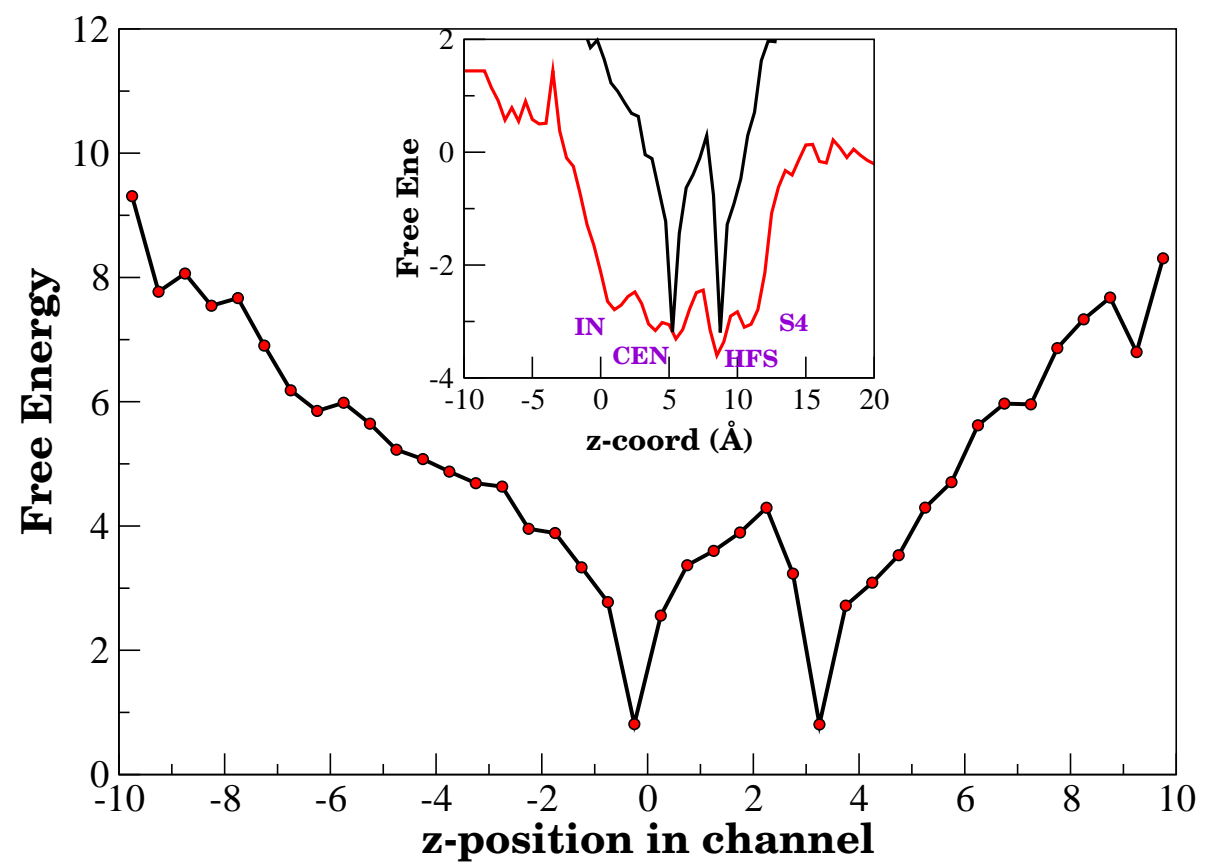

Figure 8: Potential of Mean Force of sodium in NaChBac selectivity filter computed from a 260 ns simulation of well-tempered metadynamics with axial bias on two ions in the SF. The PMF is expressed as a function of the axial distance of the ion from the center of mass of the $C_{\alpha} \mathrm{s}$ of the EEEE-ring. Inset: comparison of the PMF profiles yielded by 2D-metadynamics (black curve) and an unbiased NPT simulation (red curve). The metadynamics PMF profile has been shifted by $5.5 \AA$ along the $z$-axis to account for the position of the center of mass of the EEEE-ring with respect to the origin of the reference system. The profile has also been shifted by $4.0 \mathrm{kcal} / \mathrm{mol}$ along the vertical axis for the sake of graphical clarity. Both in the main figure and in the inset distances are expressed in Angstrom and free energies in kcal $/ \mathrm{mol}$. 


\subsection{Determination of permeation path}

The permeation mechanism of sodium in the $\mathrm{SF}$ of NaChBac was studied using two different techniques. The first method combines a Markov State Model with Transition Path Theory ${ }^{17}$ to extract the dominant permeation pathways from the trajectory of the unbiased simulation. The second method is based on identifying minimum energy paths on the axial-axial PMF derived from metadynamics using a variant of the Nudged Elastic Band algorithm. ${ }^{14}$

\subsubsection{Markov model and Transition Path Theory}

The states of the Markov model were defined as all the single, double, triple and quadruple occupancy states of the sites CC, IN, CEN, HFS, S4, EX, where IN, CEN, HFS, S4, are the binding sites in the Selectivity Filter corresponding to the minima of the Potential of Mean Force in Fig 3, CC is a region of the central cavity of the $\mathrm{NaChBac}$ channel immediately below the $\mathrm{SF}$, and $\mathrm{EX}$ is a region just above the $\mathrm{SF}$ on the extra-cellular side of the channel. Not all potential occupancy patterns were actually visited during our NPT run so that the state set comprized 43 states including 4 single occupancy states, 12 double occupancy states, 18 triple occupancy states and 9 quadruple occupancy states. In order to identify the lagtime where the dynamics of the system becomes Markovian, we computed the first 15 eigenvalues of the $43 \times 43$ matrix of transition probabilities. The eigenvalues and thus the corresponding implied timescales were computed for 40 different lagtimes (from a minimum lagtime of $20.0 \mathrm{ps}$ to a maximum lagtime of $800.0 \mathrm{ps}$ at intervals of $20.0 \mathrm{ps}$ ). The plot of implied timescales as a function of lagtime (Fig SF1 of the Supporting Information) levels out at about 200.0 ps marking the appropriate time interval for calculating the transition probability matrix that was used to determine the dominant permeation pathways.

Identification of the initial and final states of the permeation process was guided by the phenomenology exhibited by our unbiased NPT run. As already discussed, soon after the beginning of the simulation, the Selectivity Filter becomes stably occupied by two $\mathrm{Na}^{+}$ions in the CEN and HFS sites. The trajectory then shows several transient entering-events of a third sodium ion. Accordingly, the initial state is chosen as a configuration with three ions in sites CEN, HFS and EX. This configuration turned out to be the most populated occupancy state with an ion in the EX site. In a similar way the analysis of the trajectory revealed that when a $\mathrm{Na}^{+}$ion leaves the IN site to enter the Central Cavity of the channel, the SF is still occupied by two sodium ions in CEN and S4. The final state was therefore chosen as a triple occupancy state with ions in CC, CEN and S4. This arrangement is the most populated state with an ion in the CC site. The application of the Transition Path Theory methodology identified 40 pathways connecting the initial and final state. If $f_{i}$ represents the bottleneck net flux of path $i$, the relative probability with which path $i$ is used can be computed as $f_{i} / \sum_{j} f_{j}$. As illustrated in Fig SF2 of the Supporting Information, where we plot the probability of each pathway as well as the cumulative probability, the first 7 paths account for $70 \%$ of the total flux from the initial to the final state.

The seven dominant pathways are illustrated in Fig 9. From this graph it can be noted that the most probable path transits through states 28-27-20-17. This path could be merged with the one through states $28-20-17$, which is basically identical apart for the fact that it bypasses state-27. The main path starts with the initial configuration with ions in positions CEN, HFS and EX. In the next step the ion in position EX advances by one position occupying site $\mathrm{S} 4$. This apparently causes an electrostatic repulsion that pushes forward the ions in CEN and HFS until they occupy sites IN and CEN respectively. In the last step of the pathway the ion in site IN leaves the selectivity filter entering the central cavity of the ion. As a final remark, it must be stressed that the statistical sampling of the permeation events observed in our unbiased NPT run is too limited to allow quantitatively reliable conclusions. This occurs because 
our simulation was performed in the absence of any external electric field and with equal $\mathrm{NaCl}$ concentrations on the two sides of the membrane so that there was no electrochemical gradient driving permeation events. As a result, the probabilities of many of the transitions illustrated in Fig 9 were below the cutoff for printout in the sparse matrix representation employed by the MSM-Builder software. ${ }^{18}$ Yet, even if overall permeation probabilities are very low, some permeation paths do stand out as being more probable than others.

\subsubsection{Nudged Elastic Bands}

The PMF as a function of the position of the two ions in the SF can also be used to study the permeation mechanism, thus making comparisons with the permeation pathway identified through Transition Path Theory (TPT). The comparison is not straightforward since the TPT approach revealed that the most probable permeation pathway requires the presence of three ions in the SF while the metadynamics simulations were biased so that only two ions explored this region. In particular, the maximum flow path identified by TPT starts from a configuration with an ion in the EX site, just above the SF, and two other ions in the HFS and CEN sites respectively. The most similar configuration that can be attained using two ions only involves an ion above the SF and another ion in the middle of the region spanned by sites CEN and HFS. More specifically, the second ion will be placed in the upper part of site CEN, near the border with site HFS. The final state on the other hand was characterized by a ion in the central cavity, below the SF, and the other ion in site IN. In Fig 7 the two solid lines represent Minimum Energy Paths (MEP) connecting the initial and final states computed using a variant of the Nudged Elastic Band (NEB) method as detailed in Ref. ${ }^{14}$ It can be noted that the two MEP follow an approximately L-shaped profile. In the upper path the vertical branch of the $\mathrm{L}$ is characterized by a progressive approach of the ion originally lo- cated above the SF, while the second ion retains its initial position in the CEN site. When the incoming ion also reaches site CEN it becomes sufficiently close to the second ion to push it to site IN and then into the central cavity of the channel. The incoming ion will in turn proceed to site IN. The lower MEP, due to the symmetry of the PMF, is essentially identical to the upper one except for minor differences related to the residence time in the different states. Schematically, the permeation process can be described as follows and is also illustrated in Fig 10: $(C E N, E x t) \rightarrow(C E N, S 4) \rightarrow$ $(C E N, H F S) \rightarrow(C E N, C E N) \rightarrow(I N, C E N) \rightarrow$ $(C C, C E N) \rightarrow(C C, I N)$

This knock-on mechanism, as shown in Fig 10, appears to be consistent with the one identified through TPT. In the TPT-derived mechanism, when the incoming ion accesses the SF it occupies site S4 while the two internal ions sit in sites CEN and HFS. In the two-ion mechanism, since there is only one internal ion in site CEN, the incoming ion can reach a deeper position inside the SF arriving as far as site CEN. In both the TPT-derived and NEB-derived mechanisms the key event is the repulsion of the internal ion(s) due to the arrival of the incoming one. In the TPT-derived path the two internal ions are pushed to sites IN and CEN while the incoming ion remains in site S4. In the NEB-derived path the single internal ion is pushed to site IN while the incoming ion temporarily remains in site CEN. A difference between the two mechanisms appears in the last step due to the different number of ions involved. In the TPT-derived path, the two internal ions in sites IN and CEN that so far had always occupied neighboring sites split, the former moving to the internal cavity and the latter remaining in CEN. This obviously can not occur in the NEB-derived path where the single internal ion pushed in site IN simply advances into the central cavity of the channel. 


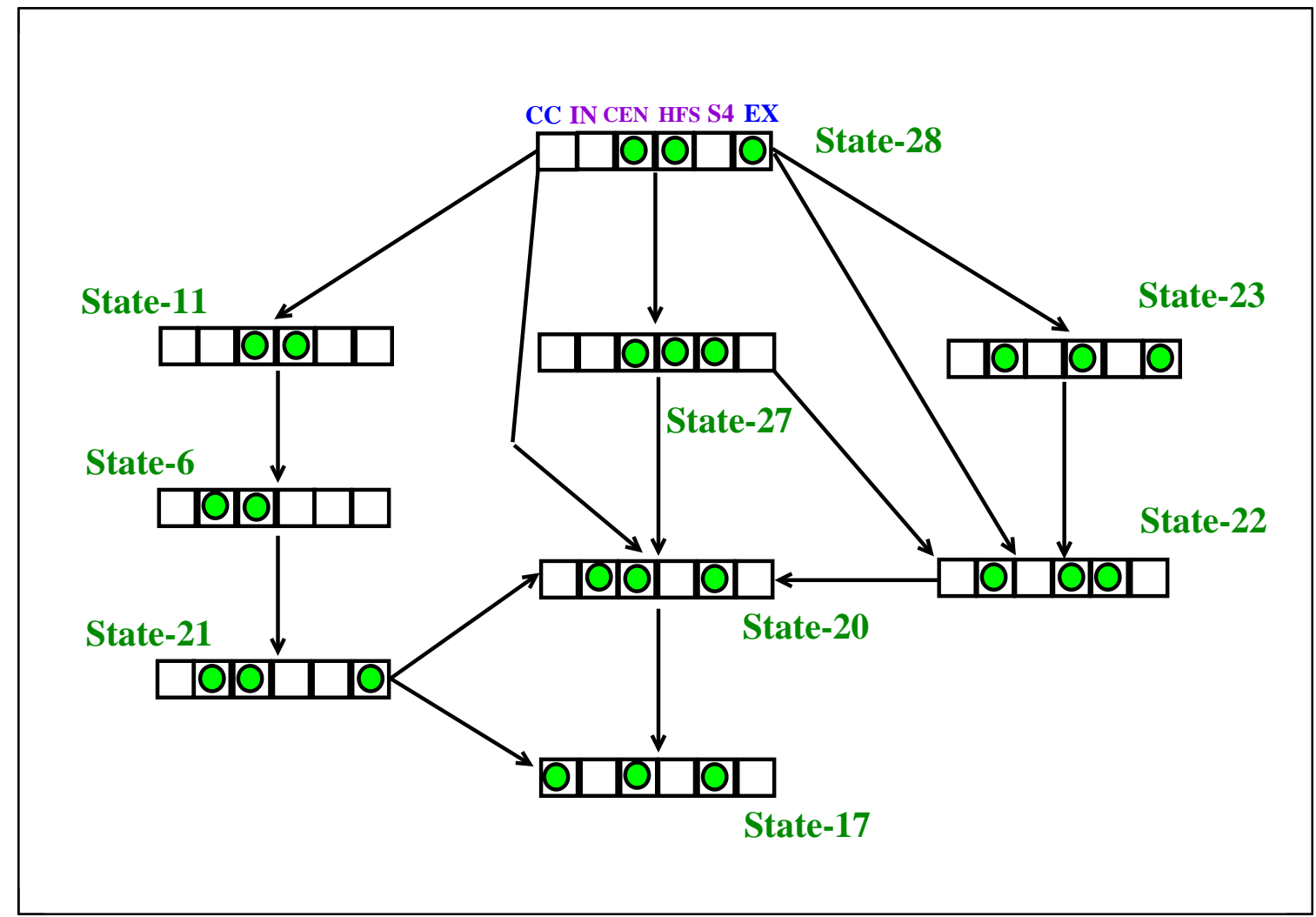

Path-1 (28-27-20-17) : 17\%

Path-2 (28-22-20-17): 12\%

Path-5 (28-11-6-21-17): 9\%

Path-6 (28-20-17): 6\%

Path-3 (28-27-22-20-17): $13 \%$

Path-7 (28-23-22-20-17): 4\%

Path-4 (28-11-6-21-20-17): 9\%

Figure 9: Dominant permeation pathways as derived from Transition Path Theory. The seven dominant paths account together for $70 \%$ of the total flux. 


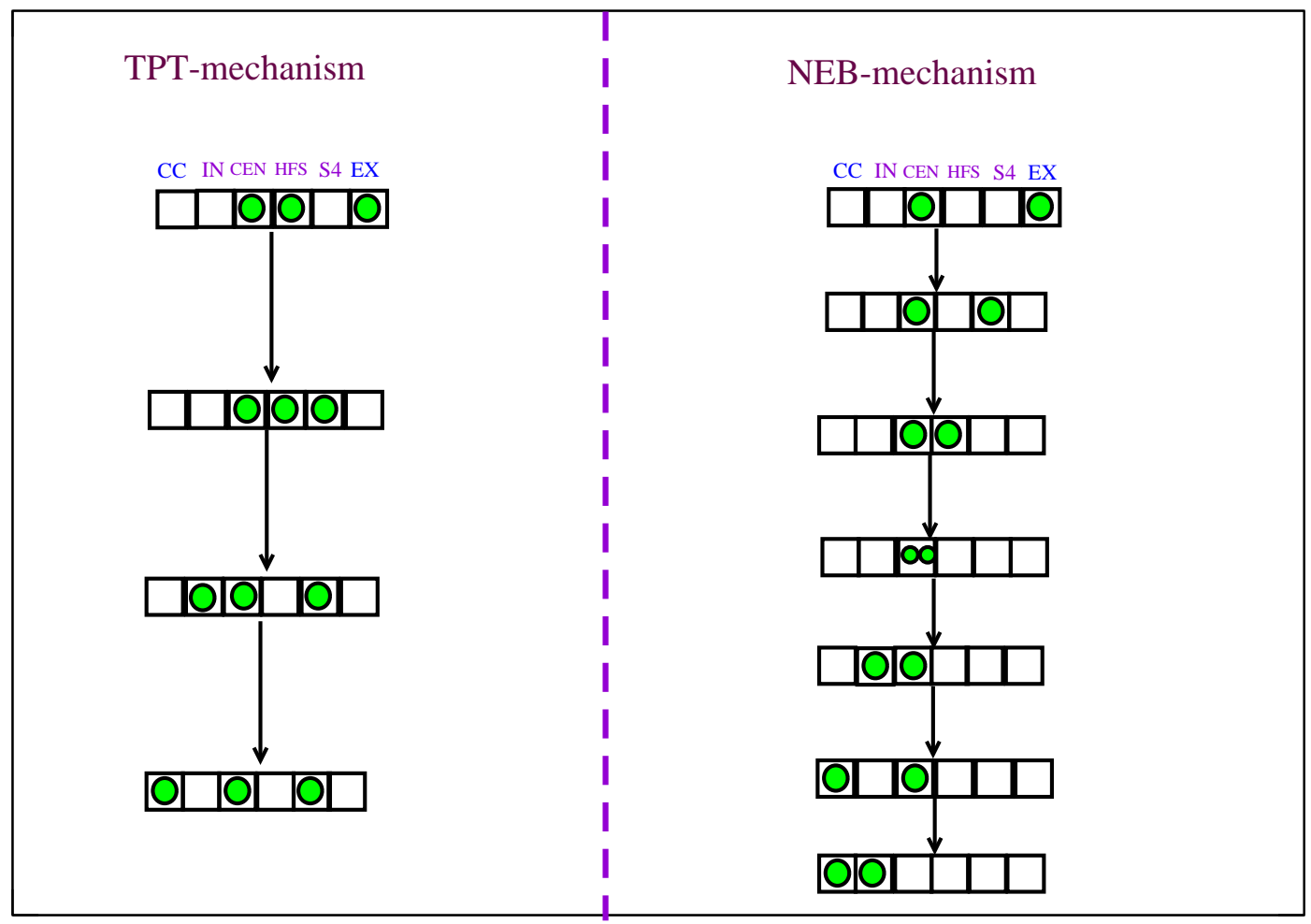

Figure 10: Comparison of $\mathrm{Na}^{+}$permeation mechanisms in the $\mathrm{NaChBac}$ channel. The first mechanism corresponds to the most probable pathway predicted by Transition Path Theory (see Fig 9). The second mechanism was determined applying a variant of the Nudged Elastic Band method to the axial-axial PMF derived from a 260 ns metadynamics run with bias on the two ion in the Selectivity Filter. 


\section{Discussion and Conclu- sions}

In this work we investigated ion transport across the NaChBac channel by means of Molecular Dynamics simulations. Our study addressed three main research problems: the number and position of binding sites in the SF, the hydration and coordination pattern of permeating $\mathrm{Na}^{+}$ions, and the characterization of the permeation mechanism. Even if $\mathrm{NaCh}-$ Bac was the first bacterial sodium channel to be identified, ${ }^{5}$ computational studies on this system were very limited ${ }^{19}$ because no crystal structure was available. We thus built an homology model based on the NavMs template.

During a long unbiased simulation water molecules and sodium ions flow spontaneously inside the SF, which is occupied by two ions during almost the entire simulation. Several transient events of access by a third ion are also observed. The Potential of Mean Force computed from the unbiased run showed the existence of four main binding sites, three of which correspond to sites IN, CEN and HFS that were predicted by Catterall and coworkers ${ }^{8}$ based on their analysis of the NavAb crystal structure. Our results are also consistent with a number of computational works on the homologous NavAb system. In agreement with the calculations of Furini and Domene ${ }^{12}$ and Stock et al ${ }^{14}$ we also found that sites CEN and HFS are the most stable binding sites while sites IN and S4 are much higher in energy and they disappear in the PMF computed from metadynamics. In agreement with the findings of Furini and Domene, our axial-radial PMF shows that in the innermost IN and CEN sites sodium tends to occupy an on-axis central position where it is surrounded by a whole hydration shell of six water molecules. Conversely, when $\mathrm{Na}^{+}$occupies the HFS site it interacts with a Glutamate and a Serine often belonging to the same subunit of the selectivity filter so that it is forced to reside in an off-axis position. The pattern of hydration and coordination might be critical for the understanding of the selectivity mechanism. Corry and Thomas ${ }^{11}$ for instance sug- gested that the scarse permeability of NavAb to $\mathrm{K}^{+}$ions could be due to the inability of this ion to coordinate water molecules in the optimal geometry required to fit inside the EEEEring. A similar mechanism was suggested by Ke et $a l^{30}$ to rationalize the patterns of $\mathrm{Na}^{+} / \mathrm{Ca}^{2+}$ selectivity in NavAb. Our results are also consistent with the findings of Chakrabarti et $a l^{34}$ who performed equilibrium multi-microsecond simulations of NavAb. These authors observed spontaneous permeation of $\mathrm{Na}^{+}$through the $\mathrm{SF}$ in a knock-on mechanism involving alternating states in which 2 or 3 ions are within the pore lumen. The frequencies of single, double and triple occupancies of the SF of NavAb (11\%, $66 \%$ and $23 \%$ ) are consistent with the corresponding occupancy rates we found in $\mathrm{NaCh}-$ Bac $(6 \%, 64 \%$ and $30 \%)$ resulting from the structural similarities of the two proteins. On account of the importance of the ions in the Central Cavity for the description of the permeation mechanism, Chakrabarti et al identified four different macrostates 1', 2, 2' and 3 where the integer value refers to the occupancy of the $\mathrm{SF}$ and the primed and unprimed states correspond to the presence or absence of $\mathrm{Na}^{+}$ions in the Central Cavity. Using these notations, two main permeation pathways were identified: $2 \rightarrow 1^{\prime} \rightarrow 2^{\prime}$ and $2 \rightarrow 3 \rightarrow 2^{\prime}$. The choice of a starting state with two ions in the SF was also employed in our work due to the high frequency of conformations with an ion in CEN and an ion in HFS. The $2 \rightarrow 3 \rightarrow 2^{\prime}$ pathway basically corresponds to the most likely path we identified through Markov Modelling and Transition Path Theory (path-1: 28-27-20-17; see Fig 9). The $2 \rightarrow 1^{\prime} \rightarrow 2^{\prime}$ path, on the other hand, has some resemblance to path- 4 and path- 5 of Fig 9 where the ions inside the SF start moving toward the Central Cavity before the third ion enters the SF. At variance with Chakrabarti's pattern, however, in our mechanism the ions in the SF never access the Central Cavity before the third ion has entered the SF. A similar permeation mechanism described as a two-stage process alternating between three and two-ion occupancy states was also recently reported in NavAb by Boiteux et al. ${ }^{33}$

Our findings are also in good agreement with 
the microsecond Molecular Dynamics simulations that Ulmschneider et $a l^{31}$ performed on the NavMs channel, the template we used to build the NaChBac homology model. As in our case, these authors performed simulations at the non physiological $0.5 \mathrm{M}$ concentration of $\mathrm{NaCl}$ to enhance the number of permeation events. For the same reason their simulations were performed at a temperature slightly higher than ours ( $310 \mathrm{~K}$ vs $300 \mathrm{~K}$ ) and in the presence of constant electric fields corresponding to voltages in the 100-500 $\mathrm{mV}$ range. Irrespective of the applied voltage, the analysis of the PMF reveals the existence of five sodium binding sites labeled S0-S4. While site S2 is a transient and scarcely populated site that does not appear in our $\mathrm{NaChBac}$ simulations, the remaining four sites $\mathrm{S} 0, \mathrm{~S} 1, \mathrm{~S} 3$ and $\mathrm{S} 4$ correspond to sites S4, HFS, CEN and IN that we identified analysing the minima of the PMF from the unbiased simulation (Fig 3). The coordination patterns and the binding positions are also similar. Both in NaChBac and NavMs simulations the ion in site $\mathrm{S} 1=\mathrm{HFS}$ directly interacts with a glutamate residue adopting an off-axis placement while in sites $\mathrm{S} 3=\mathrm{CEN}$ and $\mathrm{S} 4=\mathrm{IN}$ the ion tends to retain its full hexa-coordinated water shell occupying a more central position. The similarity of the results of the equilibrium $\mathrm{NaChBac}$ simulations and the out-of-equilibrium simulations of NavMs in the presence of an electric field, show that the mode of binding and hydration in voltage gated sodium channels is robust with respect to an applied voltage. The applied voltage however, somewhat interferes with the permeation mechanism. Ulmschneider et al in fact, did not observe a knock-on mechanism but rather single $\mathrm{Na}^{+}$passage through the SF. Nevertheless, at any time the channel was typically occupied by more than one ion with a time-averaged $1.8 \mathrm{Na}^{+}$in agreement with a value of 2.2 in our unbiased NPT simulation. In order to check the consistency of our simulations with the current-voltage plot determined by Ulmschneider et al, we exploited a collective diffusion model introduced by Liu and $\mathrm{Zhu}^{35}$ to relate the spontaneous permeation events at equilibrium with steady currents induced by small voltages. As shown in
Fig 11, the currents predicted using the Linear Response Theory exhibit values close to those reported by Ulmschneider et $a l^{31}$ suggesting a similar electrophysiological behaviour of $\mathrm{NaCh}$ Bac and NavMs. This is also confirmed by the single channel conductance of $42 \mathrm{pS}$ in NaChBac and $40 \mathrm{pS}$ in NavMs. Even if the similarity in electrophysiological behaviour of $\mathrm{NaChBac}$ and NavMs is justified by the high sequence identity, it may also be a sign that our homology model too faithfully reproduced the structural features of the NavMs template, resulting in an inability to show specific functional patterns. This is why a comparison with experimental measurements on NaChBac is a necessary benchmark. Even if a wide experimental literature does exist on $\mathrm{NaChBac}$ physiology, ${ }^{5,6}$ a quantitative comparison with our data can not be attempted, because, in order to enhance the statistics of permeation events, our simulations were run using a high $(0.5 \mathrm{M})$ concentration of $\mathrm{NaCl}$ that is not routinely reported in experimental studies. To fill this gap we performed single channel recordings of $\mathrm{NaChBac}$ in the same concentration conditions used in simulations (Fig S7 of Supporting Information). As shown in the inset of Fig 11, our electrophysiological data confirm the predictions of MD simulations thus providing an experimental validation to both the NaChBac homology model and our simulation protocol.

The permeation and selectivity properies of Calcium and Sodium channels may be rationalised within the framework of the Ionic Coulomb Blockade (ICB) model. ${ }^{28,29}$ The main tenet of this model is that permeation in these channels is mainly driven by the charge $Q_{f}$ of the Selectivity Filter. In fact, as shown in Brownian Dynamics simulations and formally predicted by the ICB model, the current does not increase monotonically with $Q_{f}$ but shows a number of peaks separated by blockade points. According to the ICB model conduction occurs when the charge $Q_{f}$ balances the charge of the ions inside the channel plus the image charge that the incoming ion induces in the channel due to polarization effects. Formally this can 


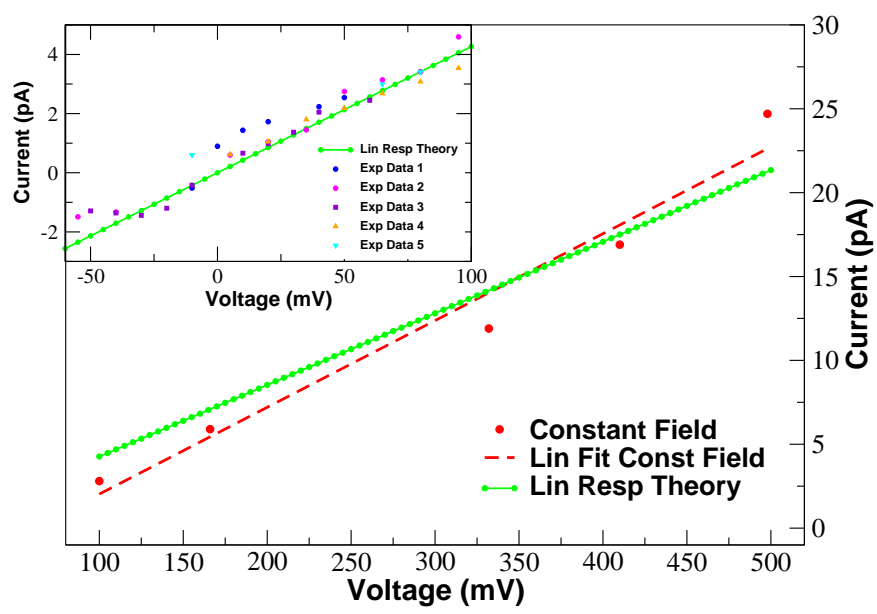

Figure 11: Stationary currents in $\mathrm{NaChBac}$ and NavMs. The red dots in the main figure correspond to the currents in NavMs computed in $\mathrm{ref}^{31}$ applying a constant electric field. The dashed line is a linear fit of these data. The green dotted line is the $\mathrm{I}-\mathrm{V}$ curve predicted from our unbiased $\mathrm{NaChBac}$ simulation using the method based on linear response theory. Inset: comparison of predictions of Linear Response Theory (green dotted line) and experimentally obtained $\mathrm{NaChBac}$ single channel current voltage relationships recorded from five independent experiments (represented by blue, magenta, violet, orange and cyan symbols) in symmetrical $0.5 \mathrm{M} \mathrm{Na}^{+}$. Error bars are not shown since they are smaller than symbol size. be expressed as: ${ }^{29}$

$$
Q_{f}(i, z)=z e / 2+z e i=z e(i+1 / 2)
$$

where $z e / 2$ is the image charge of an ion of charge ze and zei is the charge of $i$ ions already inside the SF. In more practical terms this expression means that the minimal charge that the SF should have to draw in one more ion, given that $i$ ions are already inside is $z e(i+1 / 2)$. This simple expression provides a simple and elegant explanation of the permeation patterns observed in our NaChBac simulations. When the $\mathrm{SF}$ is empty, the minimal charge required to draw in the first ion must balance the image charge of a single $\mathrm{Na}^{+}$ion, i.e. -1/2. This condition is clearly fulfilled by an empty SF whose charge is - 4 due to the charged side-chains of the glutamates of the EEEE-ring. The first sodium ion is thus pulled into the SF changing its net charge to -3 . This charge is more than sufficient to pull into the SF one more ion since Eq 1 predicts that the minimal charge the filter must have to draw in a second $\mathrm{Na}^{+}$ion when one is already inside is $-3 / 2$. The second sodium ion is thus pulled in raising the SF charge to -2 . In order to attract a third ion when two ions are already in, the SF should have at least a charge of $-5 / 2=-2.5$. The model thus predicts that a stable 3-ion arrangement can not be achieved. This appears to be in agreement with our unbiased simulations where we could observe only transient events of access of a third ion into the SF. More specifically, if a third $\mathrm{Na}^{+}$ion happens to be inside the SF, either it is quickly pushed back into the extracellular bath, or it exerts a knock-on mechanism replacing one of the two resident ions and restoring the stable 2-ion arrangement. This approach can also be used to explain the scarce selectivity of $\mathrm{NaCh}-$ Bac for calcium. In fact, the -4 charge of the empty $\mathrm{SF}$ is more than enough to balance the +1 image charge of a Calcium ion. A single Calcium ion is thus attracted into the SF changing its charge to -2 . In order to attract a second calcium ion when one is already inside, Eq 1 predicts that the charge of the SF should be at least -3. A second Calcium ion thus can not ac- 
cess the SF. This prediction is confirmed by the work of Ke $e t a l^{30}$ and by our own ongoing unbiased simulations showing that when $\mathrm{NaChBac}$ is bathed by a $\mathrm{CaCl}_{2}$ solution, a single Calcium ion remains stuck inside the SF. The predictions based on the ICB model are tabulated in Table 1.

Table 1: Analysis of $\mathrm{Na}^{+}$and $\mathrm{Ca}^{2+}$ permeation events in $\mathrm{NaChBac}$ based on the Ionic Coulomb Blockade model. The first column shows the number of sodium or calcium ions inside the $\mathrm{SF}$, the second column shows the corresponding net charge, while the third column indicates the minimal charge the SF should have to draw inside one more ion given that $i$ ions are already in. The fourth column finally shows how the number of ions inside the SF changes if the condition indicated in column 3 is satisfied by the charge tabulated in column 2 .

\begin{tabular}{|c|c|c|c|}
\hline $\mathrm{Na}^{+}$-in & $Q_{f}$ & $Q_{f}(i, 1)=(i+1 / 2)$ & Permeat \\
\hline 0 & -4 & $-1 / 2$ & $0 \rightarrow 1$ \\
\hline 1 & -3 & $-3 / 2$ & $1 \rightarrow 2$ \\
\hline 2 & -2 & $-5 / 2$ & No perm. \\
\hline \hline $\mathrm{Ca}^{2+}$-in & $Q_{f}$ & $Q_{f}(i, 2)=2(i+1 / 2)$ & Permeat \\
\hline 0 & -4 & -1 & $0 \rightarrow 1$ \\
\hline 1 & -2 & -3 & No perm. \\
\hline
\end{tabular}

The analysis of the permeation mechanism is customarily performed using an enhanced sampling technique such as Metadynamics.$^{16}$ Metadynamics is an extremely powerful technique, but it suffers from a number of limitations. In the context of our study the problem relies on the fact that the number of collective variables, i.e. the number of ions biased to explore the SF, must be chosen a priori. This means that in order to attain reliable results, a correct guess of the permeation mechanism should be available in the first place. In our case this determined the mismatch between the PMF from the unbiased simulation and the one yielded by $1 \mathrm{D}$-metadynamics. When only a single ion is biased to reside in the $\mathrm{SF}$, the PMF features a single very deep minimum at the level of site CEN. This reflects the existence of an energy barrier in the middle of the
SF that prevents a single ion from crossing the filter, in agreement with a number of computational findings ${ }^{11-14}$ on the NavAb channel. Finol-Urdaneta et $a l^{32}$ for instance, by means of one and two-dimensional Umbrella sampling simulations, showed that a single $\mathrm{Na}^{+}$or $\mathrm{K}^{+}$ ion binds the SF too tightly to allow physiological rates of throughput. On the other hand, when two monovalent cations are allowed to enter the pore, more realistic, low-energy permeation pathways become apparent. We confirmed this hypothesis by a second metadynamics simulation whereby two ions were biased to explore the SF and that produced a PMF in good agreement with the unbiased one, featuring two main minima in correspondence of sites CEN and HFS.

The axial-axial PMF yielded by 2Dmetadynamics was also used to determine a permeation mechanism defined by the sequence of the events along the Minimum Energy Path connecting two configurations before and after a permeation event. The knock-on mechanism predicted through this strategy clearly only involves two ions because in the metadynamics run only two ions were allowed to explore the SF. This however, is not sufficient to exclude a mechanism involving more ions. This is why we investigated the permeation mechanism also through a combination of Markov State Modeling and Transition Path Theory, ${ }^{17}$ an approach developed for the analysis of phenomena occurring on timescales currently not accessible with standard computational technology. In our work instead of assembling a large number of small trajectories, as it is customary, we relied on a single long one. The two approaches are equivalent provided that the aggregate simulation time is sufficiently long. As a rule of thumb, the aggregate simulation time should be at least of the same order of magnitude of the timescale of the investigated process. In our case, however, to the best of our knowledge, there is no experimental estimate of the timescale of permeation in $\mathrm{NaChBac}$, especially in the absence of any electric field. Moreover, the event defining the timescale of permeation, also varies according to the permeation mechanism envisaged. If permeation 
is a single-ion process, then its timescale is the average time taken by an ion to completely cross the SF. Using this definition for the analysis of the unbiased trajectory, we estimated a timescale of 10-12 ns which means that on average 10 permeation events should be observed in a $100 \mathrm{~ns}$ trajectory. Indeed in our simulation only one such event was observed. The scenario is different if permeation is a multi-ion event. The Mean First Passage Time from state-28 to state-17 (the most likely path shown in Fig 9) is of the order of 100 ps which means that on average 1000 permeation events should be observed in a 100 ns trajectory. This short timescale results from the fact that in a multi-ion mechanism an ion does not have to cross the whole SF. For instance, comparing State-28 and State-17 in Fig 9 it can be noted that the ion originally in site EX only advances to site S4, while the ion in HFS only moves to CEN and the ion in CEN goes a little farther to the CC region. Even if the statistics of our trajectory is not huge, it is thus reasonable. The three-ion mechanism predicted as the most likely from the Transition Path Theory, in any case is still a knock-on mechanism in agreement with the predictions of the Nudged Elastic band methodology. The two techniques therefore cross-validate each other defining a protocol that could be applied to the study of other channel systems.

\section{Supporting Information Available}

Supporting Information. (1) Potential of Mean Force Calculation; (2) Nudged Elastic Band method; (3) Markov State Model and Transition Path Theory. (4) current calculation through a collective diffusion model; (5) Patch-clamp measurements of curents in $\mathrm{NaCh}$ Bac; (6) fluctuations in $\mathrm{Na}^{+}$coordination; Supporting Information Figures: (SF1) Implied Timescale of Markov Model; (SF2) Fluxes of permeation pathways. (SF3) Sequence alignment $\mathrm{NaChBac/NavMs/NavAb. \quad (SF4)}$ Structural overlays NaChBac/NavMs, NaChBac/NavAb. (SF5) Space-filling representa- tions of NaChBac, NavMs and NavAb. (SF6) Diffusion coefficient of net transported charge. (SF7) Patch-clamp recordings. Supporting Information Tables: (ST1) Parameters of equilibration.

\section{Acknowledgments}

The authors gratefully acknowledge financial support from EPSRC (grant EP/M016889/1 and EP/M015831/1). Project: Ionic Coulomb blockade oscillations and the physical origins of permeation, selectivity, and their mutation transformations in biological ion channels. Computational facilities were provided by the MidPlus Regional Centre of Excellence for Computational Science, Engineering and Mathemtatics, under EPSRC grant EP/K000128/1, and by the University of Warwick Scientific Computing Research Technology Platform.

\section{References}

(1) Koishi, R.; Xu, H.; Ren, D.; Navarro, B.; Spiller, B.W.; Shi, Q.; Clapham, D.E. Membrane Transport, Structure, Function, and Biogenesis J. Biol. Chem. 2004, $279(10), 9532-9538$.

(2) Hofman, F.; Flockerzi, V.; Kahl, S.; Wegener, J.W. L-Type $\mathrm{Ca}_{V} 1.2$ Calcium Channels: From In Vitro Findings to In Vivo Function Physiol. Rev. 2014, 94, 303-326.

(3) Kim, J.B. Channelopathies Korean J. Pediatr. 2014, 57(1), 118.

(4) Sato, C.; Ueno, Y.; Asai, K.; Takahashi, K.; Sato, M.; Engel, A.; Fujiyoshi, Y. The voltage-sensitive sodium channel is a bellshaped molecule with several cavities $\mathrm{Na}$ ture 2001, 409, 1047-1051.

(5) Ren, D.; Navarro, B.; Xu, H.; Yue, L.; Shi, Q.; Clapham, D.E. A Prokaryotic Voltage-Gated Sodium Channel Science 2001, 294, 2372-2375. 
(6) Yue, L.; Navarro, B.; Ren, D.; Ramos, A.; Clapham, D.E. The Cation Selectivity Filter of the Bacterial Sodium Channel NaChBac J. Gen. Physiol. 2002, 120, 845-853.

(7) Roux, B. Ion Conduction and Selectivity in $\mathrm{K}^{+}$Channels Annu. Rev. Biophys. Biomol. Struct. 2005, 34, 153-171.

(8) Payandeh, J.; Scheuer, T.; Zheng, N.; Catterall, W.A. The crystal Structure of a Voltage-Gated Sodium Channel Nature 2011, 475, 353-358.

(9) Carnevale, V.; Trptow, W.; Klein, M.L. Sodium Ion Binding Sites and Hydration in the Lumen of a Bacterial Ion Channel from Molecular Dynamics Simulations $J$. Phys. Chem. Lett. 2011, 2, 2504-2508.

(10) Naylor, C.E.; Wallace, B.A.; Bagneris, C.; DeCaen, P.G.; Sula, A.; Scaglione, A.; Clapham, D.E., Molecular Basis of Ion Permeability in a Voltage-Gated Sodium Channel The EMBO J. 2016, 135, 820830.

(11) Corry, B.; Thomas, M. Mechanism of Ion Permeation and Selectivity in a Voltage Gated Sodium Channel J. Am. Chem. Soc. 2012, 134, 1840-1846.

(12) Furini, S.; Domene, C. On Conduction in a Bacterial Sodium Channel PLoS Comput. Biol. 2012, 8, e1002476.

(13) Qiu, H.; Shen, R.; Guo, W. Ion Solvation and Structural Stability in a Sodium Channel Investigated by Molecular Dynamics Calculations Biochim. Biophys. Acta 2012, 1818, 2529-2535.

(14) Stock, L.; Delemotte, L.; Carnevale, V.; Treptow, W.; Klein, M.L. Conduction in a Biological Sodium Selective Channel $J$. Phys. Chem. B 2013, 117, 3782-3789.

(15) McCusker, E.; Bagneris, C.; Naylor, C.E.; Cole, A.R.; D'Avanzo, N.; Nichols, C.G.; Wallace, B.A. Structure of a Bacterial
Voltage-Gated Sodium Channel Pore Reveals Mechanisms of Opening and Closing Nature Comm. 2012, 3(1102), 1-8.

(16) Laio, A.; Gervasio, F.L. Metadynamics: a Method to Simulate Rare Events and Reconstruct the Free Energy in Biophysics, Chemistry and Material Science Rep. Prog. Phys. 2008, 71, 126601 (22pp)

(17) Noè, F.; Schütte, C.; Vanden-Eijnden, E.; Reich, L.; Weikl, T.R. Constructing the Equilibrium Ensemble of Folding Pathways from Short Off-Equilibrium Simulations Proc. Natl. Acad. Sci. USA 2009, 106(45), 19011-19016.

(18) Beauchamp, K.A.; Bowman, G.R.; Lane, T.J.; Maibaum, L.; Haque, I.S.; Pande, V.S. MSMBuilder2: Modeling Conformational Dynamics on the Picosecond to Millisecond Scale J. Chem. Theory Comput. 2004, 7, 3412-3419.

(19) Barber, A.F.; Carnevale, V.; Raju, S.G.; Amaral, C.; Treptow, W.; Klein, M.L. Hinge-Bending Motions in the Pore Domain of a Bacterial Voltage-Gated Sodium Channel Biochim. Biophys. Acta 2012, 1818, 2120-2125.

(20) Shafrir, Y.; Durell, S.R.; Guy, H.R. Models of Voltage-dependent Conformational Changes in NaChBac Channels Biophys. J. 2008, 95, 3663-3676.

(21) McCusker, E.C.; D'Avanzo, N.; Nichols, C.G.; Wallace, B.A. Simplified Bacterial "Pore" Channel Provides Insight into the Assembly, Stability and Structure of Sodium Channels J. Biol. Chem. 2011, 286, 16386-16391.

(22) Choudhary, O.P.; Paz, A.; Adelman, J.L.; Colletier, J.P.; Abramson, J.; Grabe, M. Structure-Guided Simulations Illuminate the Mechanism of ATP Transport through VDAC1 Nat. Struct. Mol. Biol. 2014, 21(7), 626-632.

(23) Sali, A.; Blundell, T.L. Comparative Protein Modelling by Satisfaction of Spatial 
Restraints J. Mol. Biol. 1993, 234, 779815.

(24) Phillips, J.C.; Braun, R.; Wang, W.; Gumbart, J.; Tajkhorshid, E.; Villa, E.; Chipot, C.; Skeel, R.D.; Kale, L.; Schulten, K. Scalable Molecular Dynamics with NAMD J. Comput. Chem. 2005, 26, 17811802.

(25) Dickson, C.J.; Madej, B.D.; Skjevik, A.A.; Betz, R.M.; Teigen, K.; Gould, I.R.; Walker, R.C. Lipid14: The Amber Lipid Force Field J. Chem. Theory Comput. 2014, 10, 865879 .

(26) Maier, J.A.; Martinez, C.; Kasavajhala, K.; Wickstrom, L.; Hauser, K.E.; Simmerling, C. ff14SB: Improving the Accuracy of Protein Side Chain and Backbone Parameters from ff99SB J. Chem. Theory Comput. 2015, 11, 36963713.

(27) Barducci, A.; Bussi, G.; Parrinello, M. Well-Tempered Metadynamics: A smoothly Converging and Tunable FreeEnergy Method Phys. Rev. Lett. 2008, 100, 020603.

(28) Kaufman, I.K.; McClintock, P.V.E.; Eisenberg, R.S. Coulomb Blockade Model of Permeation and Selectivity in Biological Ion Channels New J. Phys. 2015 17(8), 083021.

(29) Kaufman, I.K.; Luchinsky, D.G.; Tindjong, R.; McClintock P.V.E.; Eisenberg, R.S. Energetics of Discrete Selectivity Bands and Mutation-Induced Transitions in the Calcium-Sodium Ion Channels Family Phys. Rev. E. 2013 88(5), 052712.

(30) Ke, S.; Zangerl, E.-M.; Stary-Weinzinger, A. Distinct Interactions of $\mathrm{Na}^{+}$and $\mathrm{Ca}^{2+}$ Ions with the Selectivity Filter of the Bacterial Sodium Channel $\mathrm{Na}_{v} \mathrm{Ab}$ Biochem. Biophys. Res. Comm. 2013 430, 12721276.

(31) Ulmschneider, M.B.; Bagneris, C.; McCusker, E.C.; DeCaen, P.G.; Delling, M.;
Clapham, D.E.; Ulmschneider, J.B.; Wallace, B.A. Molecular Dynamics of Ion Transport through the Open Conformation of a bacterial Voltage-Gated Sodium Channel. Proc. Natl. Acad. Sci. USA 2013 110(16), 6364-6369.

(32) Finol-Urdaneta, R.K.; Wang, Y.; Al-Sabi, A.; Zhao, C.; Noskov, S.Y.; French, R.J. Sodium Channel Selectivity and Conduction: Prokaryotes Have Devised Their Own Molecular Strategy J. Gen. Physiol. 2014 143(2), 157-171.

(33) Boiteux, C.; Vorobyov, I.; Allen, T.W. Ion Conduction and Conformational Flexibility of a Bacterial Voltage-Gated Sodium Channel. Proc. Natl. Acad. Sci. USA 2014 111(9), 3454-3459.

(34) Chakrabarti, M.; Ing, C.; Payandeh, J.; Zheng, N.; Catterall, W.A.; Pomes, R. Catalysis of $\mathrm{Na}^{+}$permeation in the Bacterial Sodium Channel $\mathrm{Na}_{v} \mathrm{Ab}$ Proc. Natl. Acad. Sci. USA 2013 110(28), 1133111336.

(35) Liu, Y.; Zhu, F. Collective Diffusion Model for Ion Conduction through Microscopic Channels Biophys. J. 2013 104, 368-376. 


\section{TOC GRAPHIC}

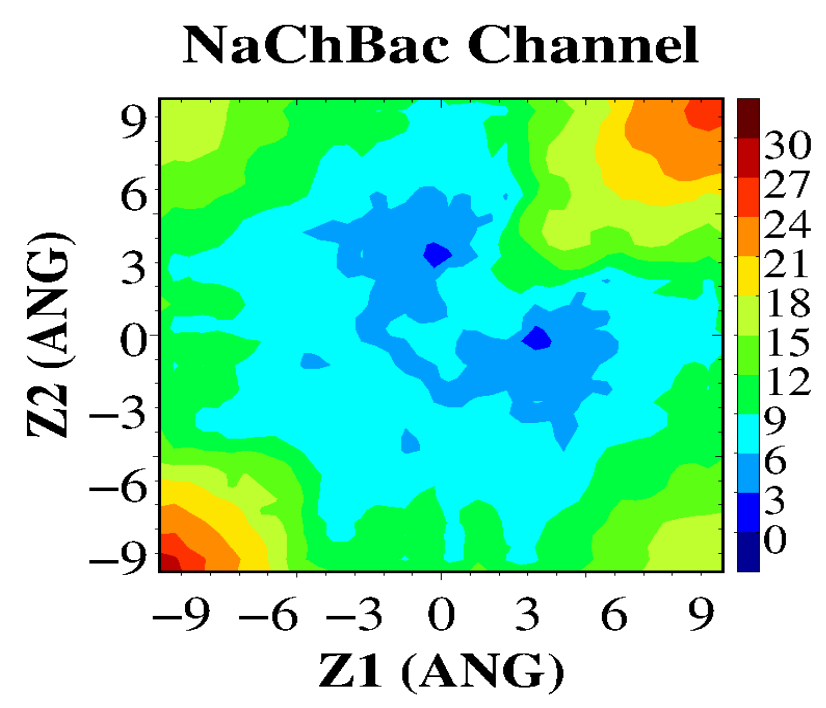

CC IN CEN HFS S4 EX

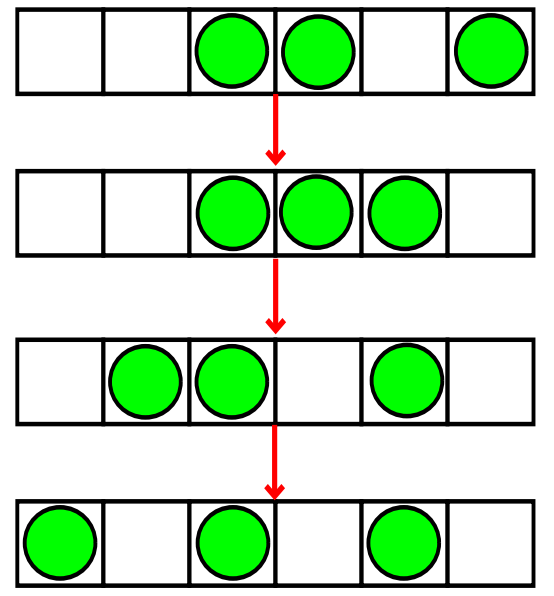

